PROCEEDINGS OF THE

AMERICAN MATHEMATICAL SOCIETY

Volume 140, Number 2, February 2012, Pages 531-539

S 0002-9939(2011)11311-X

Article electronically published on September 15, 2011

\title{
ON A RESULT OF BREZIS AND MAWHIN
}

\author{
R. MANÁSEVICH AND J. R. WARD \\ (Communicated by Yingfei Yi)
}

\begin{abstract}
Brezis and Mawhin proved the existence of at least one $T$ periodic solution for differential equations of the form$$
\left(\phi\left(u^{\prime}\right)\right)^{\prime}-g(t, u)=h(t)
$$

when $\phi:(-a, a) \rightarrow \mathbb{R}, 0<a<\infty$, is an increasing homeomorphism with $\phi(0)=0, g$ is a Carathéodory function $T$ periodic with respect to $t, 2 \pi$ periodic with respect to $u$, of mean value zero with respect to $u$, and $h \in L_{\text {loc }}^{1}(\mathbb{R})$ is $T$ periodic and has mean value zero. Their proof was partly variational. First it was shown that the corresponding action integral had a minimum at some point $u_{0}$ in a closed convex subset $\mathcal{K}$ of the space of $T$ periodic Lipschitz functions. However, $u_{0}$ may not be an interior point of $\mathcal{K}$, so it may not be a critical point of the action integral. The authors used an ingenious argument based on variational inequalities and uniqueness of a $T$ periodic solution to (0.1) when $g(t, u)=u$ to show that $u_{0}$ is indeed a $T$ periodic solution of (0.1). Here we make full use of the variational structure of the problem to obtain Brezis and Mawhin's result.
\end{abstract}

\section{INTRODUCTION}

Recently Brezis and Mawhin [1] proved the existence of at least one $T$ periodic solution for differential equations of the form

$$
\left(\phi\left(u^{\prime}\right)\right)^{\prime}-g(t, u)=h(t)
$$

when $\phi:(-a, a) \rightarrow \mathbb{R}, 0<a<\infty$, is an increasing homeomorphism with $\phi(0)=0$, $g$ is a Carathéodory function $T$ periodic with respect to $t, 2 \pi$ periodic with respect to $u$, of mean value zero with respect to $u$, and $h \in L_{l o c}^{1}(\mathbb{R})$ is $T$ periodic and has mean value zero. A special case of interest is the relativistic forced pendulum equation

$$
\left(\frac{u^{\prime}}{\sqrt{1-u^{\prime 2}}}\right)^{\prime}+A \sin (u)=h(t)
$$

The proof in 1 was partly variational. First they showed that the corresponding action integral had a minimum at some point $u_{0}$ in a closed convex subset $\mathcal{K}$ of the space of $T$ periodic Lipschitz functions. However, $u_{0}$ may not be an interior point of $\mathcal{K}$, so it may not be a critical point of the action integral. The authors then used an ingenious argument based on variational inequalities and uniqueness of a solution

Received by the editors October 17, 2010.

2010 Mathematics Subject Classification. Primary 34C25, 49J40, 58Exx.

The first author was partially supported by Fondap and Basal-CMM grants and Milenio grant P05-004F. 
to (1.1) when $g(t, u)=u$ to show that $u_{0}$ is indeed a $T$ periodic solution of (1.1). Here we make full use of the variational structure of the problem to obtain Brezis and Mawhin's result. We rewrite the problem as a two dimensional Hamiltonian system and obtain a solution by applying the now classical saddle point theorem of Rabinowitz [3] to a sequence of approximating problems, thereby obtaining a sequence of critical points. A subsequence of these critical points converges to a solution.

The reader is referred to [1] and its bibliography for a history of the $T$ periodic problem for equations (1.1), (1.2) and for the classical forced pendulum.

We first rewrite (1.1) as a system in Hamiltonian form. Let $\psi:=\phi^{-1}$ so that $\psi$ is an increasing homeomorphism from $\mathbb{R}$ onto $(-a, a)$. Equation (1.1) is equivalent to the system

$$
\begin{aligned}
& \dot{u}=\psi(v), \\
& \dot{v}=g(t, u)+h(t),
\end{aligned}
$$

where $\dot{u}=d u / d t, \dot{v}:=d v / d t$. Let $\Psi(v):=\int_{0}^{v} \psi(s) d s, G(t, u):=\int_{0}^{u} g(t, s) d s$, $w:=(u, v)$ and

$$
H(t, w):=-\Psi(v)+G(t, u)+h(t) u .
$$

System (1.3) is equivalent to

$$
\dot{w}=J \nabla_{w} H(t, w),
$$

where

$$
J=\left(\begin{array}{cc}
0 & -1 \\
1 & 0
\end{array}\right) .
$$

We will show that (1.4), and hence (1.1), has a $T$ periodic solution. We make the following assumptions:

$\left(H_{\phi}\right) \phi:(-a, a) \rightarrow \mathbb{R}$ is an increasing homeomorphism with $\phi(0)=0$, with inverse $\psi:=\phi^{-1}$.

$\left(H_{g}\right) g: \mathbb{R} \times \mathbb{R} \rightarrow \mathbb{R},(t, u) \mapsto g(t, u)$, is a bounded Carathéodory function, so $g$ is continuous in $u$ for a.a. $t$ and is Lebesgue measurable in $t$ for all $u$. Additionally, $g$ is $T$ periodic in $t$ for all $u$ and $2 \pi$ periodic in $u$ for a. $a$. $t \in \mathbb{R}$ and is of mean value zero with respect to $u$. It follows there is a constant $c_{1}$ such that both $|g(t, u)| \leq c_{1}$ and $|G(t, u)| \leq c_{1}$ for a.a. $t$ and all $u$.

Let $L_{T}^{1}$ denote the set of all $h: \mathbb{R} \rightarrow \mathbb{R}$, locally integrable and $T$ periodic. For $h \in L_{T}^{1}$ let $\bar{h}:=\frac{1}{T} \int_{0}^{T} h(s) d s$ denote the mean value of $h$.

By a (classical) $T$ periodic solution to (1.1) is meant a $T$ periodic function $u \in C^{1}(\mathbb{R}, \mathbb{R})$ such that $\phi\left(u^{\prime}\right)$ is absolutely continuous and (1.1) is satisfied a.e. on $\mathbb{R}$.

Theorem 1.1. Let $\left(H_{\phi}\right)$ and $\left(H_{g}\right)$ hold. Then (1.1) has a $T$ periodic solution for any $h \in L_{T}^{1}$ with $\bar{h}=0$.

In the next section we describe in detail our approach. In the third section we prove Theorem 1.1. 


\section{Preliminaries}

Let $E:=W_{T}^{1,2}\left(\mathbb{R}, \mathbb{R}^{2}\right)$ denote the Hilbert space of $T$ periodic $\mathbb{R}^{2}$ valued functions $w$ such that the components of $w$ are absolutely continuous and $w^{\prime} \in L^{2}\left((0, T), \mathbb{R}^{2}\right)$ with inner product

$$
\langle w, z\rangle:=\int_{0}^{T} w \cdot z d t+\int_{0}^{T} \dot{w} \cdot \dot{z} d t
$$

where $\dot{w}=d w / d t, \dot{z}=d z / d t$, and . denotes the dot product in $\mathbb{R}^{2}$. We will denote the associated norm for $w \in E$ by $\|w\|_{1}$. An equivalent norm is $\|w\|_{0}:=$ $|w|_{0}+\left(\int_{0}^{T}|\dot{w}|^{2} d t\right)^{1 / 2}$, where $|w|_{0}$ is the maximum norm in $C_{T}^{0}$, the space of continuous $T$ periodic functions. Here, for $V=\left(v_{1}, v_{2}\right) \in \mathbb{R}^{2},|V|^{2}=V \cdot V$. For $w=\left(w_{1}, w_{2}\right)$

$$
|w|_{0}:=\max _{t \in[0, T]}\left|w_{1}(t)\right|+\max _{t \in[0, T]}\left|w_{2}(t)\right| .
$$

It is known that $E$ is compactly imbedded in $C_{T}^{0}$.

For functions $w=(u, v), z=(p, q) \in E$ let

$$
\langle L w, z\rangle:=\int_{0}^{T}-J \dot{w} \cdot z d t=\int_{0}^{T} \dot{v} p-\dot{u} q d t .
$$

One can show that there is a constant $C$ such that for all such $w, z \in E$,

$$
\left|\int_{0}^{T} \dot{v} p d t\right| \leq C\|w\|_{1}\|z\|_{1} \text { and }\left|\int_{0}^{T} \dot{u} q d t\right| \leq C\|w\|_{1}\|z\|_{1},
$$

and hence (2.2) defines a bounded bilinear form on $E$ and thus defines $L$ as a bounded linear operator on $E$. The operator $L$ is self-adjoint on $E$.

The space $E$ can be written as the direct sum $E^{+} \oplus E^{0} \oplus E^{-}$of the three subspaces $E^{+}, E^{-}$, and $E^{0}$ associated with the positive, negative, and zero eigenvalues, respectively, of $L$. These eigenvalues are also the eigenvalues of the problem

$$
\dot{w}=\lambda J w, w(0)=w(T) .
$$

The eigenvalues are the set $\left\{\lambda_{n}=2 n \pi / T \mid n \in \mathbb{Z}\right\}$. The operator $L$ is positive definite on $E^{+}$, negative definite on $E^{-}$, and 0 on $E^{0}$. Note that $E^{0}$ consists of the constant vectors. Moreover, if we write $w \in E$ as $w=w^{+}+w^{0}+w^{-}$, where $w^{s} \in E^{s}, s \in\{+, 0,-\}$, then

$$
\langle L w, w\rangle=\left\langle L w^{+}, w^{+}\right\rangle+\left\langle L w^{-}, w^{-}\right\rangle,
$$

and a norm on $E$ equivalent to $\|.\|_{0}$ and $\|\cdot\|_{1}$ is given by $\|w\|^{2}=\left|w^{0}\right|^{2}+\left\langle L w^{+}, w^{+}\right\rangle-$ $\left\langle L w^{-}, w^{-}\right\rangle$. Thus we may write for $w \in E$

$$
\|w\|^{2}=\left|w^{0}\right|^{2}+\left\|w^{+}\right\|^{2}+\left\|w^{-}\right\|^{2} \text { and }\langle L w, w\rangle=\left\|w^{+}\right\|^{2}-\left\|w^{-}\right\|^{2},
$$

since the subspaces are orthogonal under the bilinear form (2.2); indeed, they are also orthogonal under the original inner product (2.1). Moreover, there are constants $m_{1}$ and $m_{2}$ such that

$$
m_{1}\|w\| \leq\|w\|_{0} \leq m_{2}\|w\| .
$$


For $w \in E$ let

$$
\mathcal{H}(w):=\int_{0}^{T} H(t, w) d t
$$

and

$$
I(w):=\frac{1}{2}\langle L w, w\rangle-\mathcal{H}(w) .
$$

We shall show, under the assumptions above, that $I$ has a critical point in $E$. This critical point will be a $T$ periodic solution to (1.1). We will need the following generalized Palais-Smale condition:

$(P S)_{c}$ A Palais-Smale sequence at level $c \in \mathbb{R}$ for $I$ is a sequence $\left\{y_{n}\right\} \subset E$ such that

$$
I\left(y_{n}\right) \rightarrow c \text { and } I^{\prime}\left(y_{n}\right) \rightarrow 0 \text { as } n \rightarrow \infty .
$$

The function $I$ satisfies the local Palais-Smale $(P S)_{c}$ condition at $c$ if the existence of a Palais-Smale sequence at level $c$ for $I$ implies that $c$ is a critical value for $I$.

We will use the following saddle point theorem, due to Rabinowitz [3] (see also 4). Originally proven with the standard Palais-Smale condition on $I$, it also holds with only $(P S)_{c}$ assumed (see [2], Theorem 4.7).

Theorem 2.1. Let $E$ be a real Banach space such that $E=E_{1} \oplus E_{2}$ with $E_{1}$ finite dimensional. Suppose $I \in C^{1}(E, \mathbb{R})$ and

(1) There is a real number $\alpha$ such that $\left.I\right|_{E_{2}} \geq \alpha$.

(2) There is a real number $\beta<\alpha$ and a bounded neighborhood $\Omega$ of 0 in $E_{1}$ such that $\left.I\right|_{\partial \Omega} \leq \beta$.

$$
\text { Let } S:=\{g \in C(\bar{\Omega}, E) \mid g(w)=w \text { for } w \in \partial \Omega\}, \text { and let } c=\inf _{g \in S} \max _{w \in \bar{\Omega}} I(g(w)) \text {. }
$$
Suppose

(3) I satisfies the local Palais-Smale condition, $(P S)_{c}$, at c. Then $c$ is a critical value for $I$.

If the conditions of Theorem 2.1 are satisfied, it follows from finite dimensional degree theory that $0 \in g(\Omega)$ for every $g \in S$. Thus for every $g \in S, \max _{w \in \bar{\Omega}} I(g(w)) \geq$ $I(0) \geq \alpha\left(\right.$ since $\left.0 \in E_{2}\right)$. Hence $c \geq \alpha$ and $c$ is finite.

\section{Proof of Theorem 1.1}

The functional $I$ in (2.6) is strongly indefinite, and Theorem 2.1 does not apply to it. We will prove Theorem 1.1 by using a modified sequence of functionals on a sequence of subspaces of $E$, to which Theorem 2.1 does apply, thus obtaining a sequence of critical points. We then show that this sequence of critical points has a convergent subsequence, the limit of which is then necessarily a weak periodic solution of (1.4). Recall that a weak periodic solution of (1.4) is a function $w=$ $(u, v) \in E$ such that for all $z=(p, q) \in E$ one has

$$
\int_{0}^{T}[\dot{v} p-\dot{u} q] d t+\int_{0}^{T}[\psi(v) q-g(t, u) p-h(t) p] d t=0 .
$$

Standard regularity arguments show that a weak periodic solution of (1.4) is a classical periodic solution of (1.4). We proceed. 
Let $E_{N}^{-}$be the subspace of $E^{-}$spanned by the eigenfunctions of $L$ associated with the first $N$ negative eigenvalues $\left\{\lambda_{n}:-N \leq n \leq-1\right\}$. Let $E_{N}:=E_{N}^{-} \oplus E^{0} \oplus E^{+}$. The space $E_{N}$ is a Hilbert space, with the same inner product as $E$. Let $P_{N}$ be the natural orthogonal projection of $E$ onto $E_{N}$ (note that for $w \in E, P_{N} w$ is represented by the truncated Fourier series of $w$, the terms associated with the eigenvalues $\left\{\lambda_{n} \mid n \leq-N-1\right\}$ omitted). Define $I_{N}$ by

$$
I_{N}(w):=\frac{1}{2}\left\langle P_{N} L w, w\right\rangle-\mathcal{H}(w)
$$

for $w \in E_{N}$. Thus

$$
I_{N}(w)=\frac{1}{2}\left\|w^{+}\right\|^{2}-\frac{1}{2}\left\|w_{N}^{-}\right\|^{2}+\int_{0}^{T} \Psi(v)-G(t, u)-h(t) u d t,
$$

where $w=(u, v)$, written as a pair, and $w=w_{N}^{-}+w_{0}+w^{+}$, where $w_{N}^{-} \in E_{N}^{-}$, $w_{0} \in E^{0}$, and $w^{+} \in E^{+}$.

We first show that conditions (1) and (2) in Theorem 2.1 hold on some subspaces $E_{1}, E_{2}$ with $E_{N}=E_{1} \oplus E_{2}$. We let $E_{1}:=E_{N}^{-}$, and $E_{2}:=E^{0} \oplus E^{+}$so that $E_{1}$ is finite dimensional and $E_{N}=E_{1} \oplus E_{2}$. Let $w_{2}=(u, v) \in E_{2}$ with $w_{2}=w^{0}+w^{+}$. We have, since $\Psi \geq 0$ and $\bar{h}=0$,

$$
\begin{aligned}
I_{N}\left(w_{2}\right) & =\frac{1}{2}\left\|w^{+}\right\|^{2}+\int_{0}^{T} \Psi\left(v^{0}+v^{+}\right)-G\left(t, u^{0}+u^{+}\right)-h(t) u^{+} d t \\
& \geq \frac{1}{2}\left\|w^{+}\right\|^{2}-c_{1} T-|h|_{L^{1}}\left|u^{+}\right|_{0} \\
& \geq \frac{1}{2}\left\|w^{+}\right\|^{2}-c_{1} T-|h|_{L^{1}} m_{2}|| w^{+} \| \\
& \geq-\frac{1}{2}|h|_{L^{1}}^{2} m_{2}^{2}-c_{1} T:=\alpha,
\end{aligned}
$$

where $m_{2}$ is a constant such that $\|w\|_{0} \leq m_{2}\|w\|$ for any $w=(u, v) \in E$. Recall that $|\cdot|_{0}$ denotes the maximum norm on $[0, T]$. For $w=w_{N}^{-}=\left(u_{N}^{-}, v_{N}^{-}\right) \in E_{1}$ we have

$$
I_{N}\left(w_{N}^{-}\right)=-\frac{1}{2}\left\|w_{N}^{-}\right\|^{2}+\int_{0}^{T} \Psi\left(v_{N}^{-}\right)-G\left(t, u_{N}^{-}\right)-h(t) u_{N}^{-} d t .
$$

Now $|\psi(v)| \leq a$ for all $v \in \mathbb{R}$, so

$$
\Psi(v)=\int_{0}^{v} \psi(s) d s \leq\left|\int_{0}^{v}\right| \psi(s)|d s| \leq a|v| .
$$

Therefore for any $\beta<\alpha$,

$$
\begin{aligned}
I_{N}\left(w_{N}^{-}\right) & \leq-\frac{1}{2}\left\|w_{N}^{-}\right\|^{2}+a \int_{0}^{T}\left|v_{N}^{-}(t)\right|-G\left(t, u_{N}^{-}(t)\right)-h(t) u_{N}^{-}(t) d t \\
& \leq-\frac{1}{2}\left\|w_{N}^{-}\right\|^{2}+a T\left|v_{N}^{-}\right|_{0}+c_{1} T+|h|_{L^{1}}\left|u_{N}^{-}\right|_{0} \\
& \leq-\frac{1}{2}\left\|w_{N}^{-}\right\|^{2}+a T m_{2}\left\|w_{N}^{-}\right\|+c_{1} T+|h|_{L^{1}} m_{2}\left\|w_{N}^{-}\right\| \\
& =-\frac{1}{2}\left\|w_{N}^{-}\right\|^{2}+\left(a T m_{2}+|h|_{L^{1}} m_{2}\right)\left\|w_{N}^{-}\right\|+c_{1} T \\
& \leq \beta<\alpha,
\end{aligned}
$$


provided $\left\|w_{N}^{-}\right\|=R$ for $R$ sufficiently large (the minimum value needed for $R$ is independent of $N$ and is easily calculated as a function of $\beta, a, c_{1}, m_{2}, T$, and $|h|_{L^{1}}$ ). This shows that (2) of Theorem 2.1 holds, with $\Omega$ the open ball in $E_{1}$ of radius $R$, centered at 0 .

We now proceed to show that $I_{N}$ satisfies $(P S)_{c}$ at every value of $c \in \mathbb{R}$. Let $c \in \mathbb{R}$ be fixed. Suppose $\left\{w_{k}\right\} \subset E_{N}, I_{N}\left(w_{k}\right) \rightarrow c$ and $I_{N}^{\prime}\left(w_{k}\right) \rightarrow 0$ as $k \rightarrow \infty$. Now for $w=(u, v), z=(p, q) \in E_{N}$, for simplicity of notation, we will omit the subscript $N$ in $w_{N, k}, w_{N, k}^{+}$, etc., and just write $w_{k}, w_{k}^{+}, \ldots$, etc., respectively. We have

$$
\begin{aligned}
I_{N}\left(w_{k}\right) & =\frac{1}{2}\left\langle P_{N} L w_{k}, w_{k}\right\rangle-\mathcal{H}\left(w_{k}\right) \\
& =\frac{1}{2}\left\|w_{k}^{+}\right\|^{2}-\frac{1}{2}\left\|w_{k}^{-}\right\|^{2}+\int_{0}^{T} \Psi\left(v_{k}\right)-G\left(t, u_{k}\right)-h(t) u_{k} d t,
\end{aligned}
$$

and for $z \in E_{N}, z=(p, q)$, we get

$$
I_{N}^{\prime}\left(w_{k}\right) z=\left\langle P_{N} L w_{k}, z\right\rangle+\int_{0}^{T} \psi\left(v_{k}\right) q-g\left(t, u_{k}\right) p-h(t) p d t .
$$

Thus letting $w_{k}=\left(u_{k}, v_{k}\right), w_{k}^{+}=\left(u_{k}^{+}, v_{k}^{+}\right)$, and $w_{k}^{-}=\left(u_{k}^{-}, v_{k}^{-}\right)$, we have

$$
\begin{aligned}
I_{N}^{\prime}\left(w_{k}\right) w_{k}^{+} & =\left\langle P_{N} L w_{k}, w_{k}^{+}\right\rangle+\int_{0}^{T} \psi\left(v_{k}\right) v_{k}^{+}-g\left(t, u_{k}\right) u_{k}^{+}-h(t) u_{k}^{+} d t \\
& =\left\|w_{k}^{+}\right\|^{2}+\int_{0}^{T} \psi\left(v_{k}\right) v_{k}^{+}-g\left(t, u_{k}\right) u_{k}^{+}-h(t) u_{k}^{+} d t \\
& \geq\left\|w_{k}^{+}\right\|^{2}-a \int_{0}^{T}\left|v_{k}^{+}\right| d t-c_{1} \int_{0}^{T}\left|u_{k}^{+}\right| d t-\|h\|_{L^{1}}\left|u_{k}^{+}\right|_{0} \\
& \geq\left\|w_{k}^{+}\right\|^{2}-C_{1}\left\|w_{k}^{+}\right\|-C_{2}\left\|w_{k}^{+}\right\|-C_{3}\left\|w_{k}^{+}\right\| \\
& =\left\|w_{k}^{+}\right\|^{2}-C_{4}\left\|w_{k}^{+}\right\|
\end{aligned}
$$

since $|\psi(v)| \leq a$ and $|g(t, u)| \leq c_{1}$, and where $C_{i}, i=1,2,3$ are constants involving imbedding and other constants and $C_{4}=C_{1}+C_{2}+C_{3}$. Since $I_{N}^{\prime}\left(w_{k}\right) \rightarrow 0$ we may assume $\left\|I_{N}^{\prime}\left(w_{k}\right)\right\| \leq 1$, and hence we get

$$
\left\|w_{k}^{+}\right\| \geq\left|I_{N}^{\prime}\left(w_{k}\right) w_{k}^{+}\right| \geq I_{N}^{\prime}\left(w_{k}\right) w_{k}^{+} \geq\left\|w_{k}^{+}\right\|^{2}-C_{4}\left\|w_{k}^{+}\right\|,
$$

from which we deduce that there is a constant $C_{5}$ such that

$$
\left\|w_{k}^{+}\right\| \leq C_{5} \text { for all } k \geq 1 \text {. }
$$

In a similar manner one can see that there is a constant $C_{6}$ such that

$$
\left\|w_{k}^{-}\right\| \leq C_{6} \text { for all } k \geq 1
$$


Now we have to deal with $\left\{w_{k}^{0}\right\}$. Let $w_{k}^{0}=\left(u_{k}^{0}, v_{k}^{0}\right) \in \mathbb{R}^{2}$. Then since $\bar{h}=0$ we have

$$
\begin{aligned}
I_{N}\left(w_{k}\right) & =\frac{1}{2}\left\|w_{k}^{+}\right\|^{2}-\frac{1}{2}\left\|w_{k}^{-}\right\|^{2}+\int_{0}^{T} \Psi\left(v_{k}^{0}+v_{k}^{+}+v_{k}^{-}\right)-G\left(t, u_{k}\right)-h(t)\left(u_{k}^{+}+u_{k}^{-}\right) d t \\
& \geq \int_{0}^{T} \Psi\left(v_{k}^{0}+v_{k}^{+}+v_{k}^{-}\right) d t-C
\end{aligned}
$$

since ||$w_{k}^{ \pm} \|$are bounded and $|G(t, u)| \leq c_{1}$. Now $\Psi(v)=\int_{0}^{v} \psi(s) d s$ and $\psi$ is increasing with $\psi(s) s>0$ for $s \neq 0$, so

$$
\lim _{|v| \rightarrow \infty} \Psi(v)=\infty
$$

and hence if $\left\{v_{k}^{0}\right\}$ is not a bounded sequence, then since $\left\|v_{k}^{+}\right\|$and $\left\|v_{k}^{-}\right\|$are bounded, we have

$$
\limsup _{n \rightarrow \infty} \int_{0}^{T} \Psi\left(v_{k}^{0}+v_{k}^{+}+v_{k}^{-}\right) d t=\infty,
$$

which contradicts (3.1) and the boundedness of $I_{N}\left(w_{k}\right)$. Therefore $\left\{v_{k}^{0}\right\}$ is bounded. Now consider $\left\{u_{k}^{0}\right\}$. These terms appear in (3.1) only in $G$. Now $G(t, u+2 j \pi)=$ $G(t, u)$ for all $u$ and all $j \in \mathbb{Z}$. Thus we can choose $\widehat{u}_{k}^{0} \in[0,2 \pi)$ with $u_{k}^{0}-\widehat{u}_{k}^{0}=2 j \pi$ for some $j \in \mathbb{Z}$, and $G\left(t, u_{k}^{0}+u_{k}^{+}(t)+u_{k}^{-}(t)\right)=G\left(t, \widehat{u}_{k}^{0}+u_{k}^{+}(t)+u_{k}^{-}(t)\right)$ for all $k \in \mathbb{N}$. Now let $y_{k}^{0}:=\left(\widehat{u}_{k}^{0}, v_{k}^{0}\right), y_{k}^{+}:=w_{k}^{+}, y_{k}^{-}:=w_{k}^{-}$and $y_{k}:=y_{k}^{0}+y_{k}^{+}+y_{k}^{-}$. Then $\left\{y_{k}\right\}$ is bounded in $E_{N}$ and $I_{N}\left(y_{k}\right)=I_{N}\left(w_{k}\right), I_{N}^{\prime}\left(y_{k}\right)=I_{N}^{\prime}\left(w_{k}\right)$ for all $k \in \mathbb{N}$. A subsequence of $\left\{y_{k}\right\}$ converges weakly in $E_{N}$ to some $y^{*} \in E_{N}$. Standard arguments (see, e.g., 2, [4) now show that the subsequence actually converges strongly in $E_{N}$ to $y^{*}$. Relabel the convergent subsequence as $\left\{y_{k}\right\}$. We have $I\left(y_{k}\right) \rightarrow c, I^{\prime}\left(y_{k}\right) \rightarrow 0$ as $k \rightarrow \infty$, and by the $C^{1}$ continuity of $I_{N}, I_{N}\left(y^{*}\right)=c$ and $I_{N}^{\prime}\left(y^{*}\right)=0$. Thus $I_{N}$ satisfies $(P S)_{c}$ at every $c \in \mathbb{R}$. By Theorem 2.1, $I_{N}$ has a critical point $w_{N} \in E_{N}$. We now need to show that the set of critical points $\left\{w_{N}: N \in \mathbb{N}\right\} \subset E$ has a convergent subsequence, converging to a weak solution of (1.3).

Let $w_{N}=\left(u_{N}, v_{N}\right)$ be a critical point for $I_{N}$. We also write $w_{N}=w_{N}^{-}+w_{N}^{0}+w_{N}^{+}$. Then for any $z \in E$, with $z_{N}=P_{N} z, z_{N}=\left(p_{N}, q_{N}\right)$, we have

$$
0=I_{N}^{\prime}\left(w_{N}\right) P_{N} z=\left\langle L w_{N}, P_{N} z\right\rangle+\int_{0}^{T}\left[\psi\left(v_{N}\right) q_{N}-g\left(t, u_{N}\right) p_{N}-h(t) p_{N}\right] d t .
$$

Hence

$$
0=I_{N}^{\prime}\left(w_{N}\right) w_{N}^{+}=\left\langle L w_{N}, w_{N}^{+}\right\rangle+\int_{0}^{T}\left[\psi\left(v_{N}\right) v_{N}^{+}-g\left(t, u_{N}\right) u_{N}^{+}-h(t) u_{N}^{+}\right] d t,
$$

and hence

$$
\begin{aligned}
\left\|w_{N}^{+}\right\|^{2}+\int_{0}^{T} & {\left[\psi\left(v_{N}\right) v_{N}^{+}-g\left(t, u_{N}\right) u_{N}^{+}-h(t) u_{N}^{+}\right] d t=0, } \\
\left\|w_{N}^{+}\right\|^{2} & \leq a T\left|v_{N}^{+}\right|_{0}+c_{1} T\left|u_{N}^{+}\right|_{0}+|h|_{L^{1}}\left|u_{N}^{+}\right|_{0} \\
& \leq\left(a T+c_{1} T+|h|_{L^{1}}\right)\left\|w_{N}^{+}\right\| .
\end{aligned}
$$

Therefore for all $N \in \mathbb{N}$

Similarly

$$
\left\|w_{N}^{+}\right\| \leq\left(a T+c_{1} T+|h|_{L^{1}}\right) .
$$

$$
\left\|w_{N}^{-}\right\| \leq\left(a T+c_{1} T+|h|_{L^{1}}\right) .
$$


As for $w_{N}^{0}=\left(u_{N}^{0}, v_{N}^{0}\right)$, we may take $u_{N}^{0} \in[0,2 \pi)$, so the sequence $\left\{u_{N}^{0}\right\}$ is bounded, and

$$
\begin{aligned}
0 & =I_{N}^{\prime}\left(w_{N}\right) w_{N}^{0}=\int_{0}^{T}\left[\psi\left(v_{N}\right) v_{N}^{0}-g\left(t, u_{N}\right) u_{N}^{0}-h(t) u_{N}^{0}\right] d t \\
& =\int_{0}^{T}\left[\psi\left(v_{N}^{0}+v_{N}^{+}+v_{n}^{-}\right) v_{N}^{0}-g\left(t, u_{N}\right) u_{N}^{0}\right] d t
\end{aligned}
$$

Since $v_{N}^{+}$and $v_{n}^{-}$are bounded, if a subsequence $\left\{v_{N_{k}}^{0}\right\}$ converges to $+\infty$, we have $\psi\left(v_{N_{k}}^{0}+v_{N_{k}}^{+}(t)+v_{N_{k}}^{-}(t)\right) \geq a / 2$ for all $k$ sufficiently large and all $t$, and then $\psi\left(v_{N_{k}}^{0}+v_{N_{k}}^{+}(t)+v_{N_{k}}^{-}(t)\right) v_{N_{k}}^{0} \rightarrow \infty$ as $k \rightarrow \infty$, uniformly on [0,T], contradicting (3.2). In the same manner, no subsequence of $\left\{v_{N}^{0}\right\}$ can diverge to $-\infty$. Thus $\left\{v_{N}^{0}\right\}$ is bounded. It follows that there is a uniform bound in $E$ on the sequence $\left\{w_{N}\right\}$. This sequence thus has a weakly convergent subsequence in $E$, strongly convergent in $C\left([0, T], \mathbb{R}^{2}\right)$, to some $w^{*} \in E$. We relabel this weakly convergent subsequence as $\left\{w_{N}\right\}$. For $z=(p, q) \in E$

$$
\left\langle L w_{N}, P_{N} z\right\rangle=-\int_{0}^{T}\left[\psi\left(v_{N}\right) q_{N}-g\left(t, u_{N}\right) p_{N}-h(t) p_{N}\right] d t .
$$

Now $L$ is self-adjoint, so $\left\langle L w_{N}, P_{N} z\right\rangle=\left\langle w_{N}, L P_{N} z\right\rangle=\left\langle w_{N}, P_{N} L z\right\rangle=\left\langle w_{N}, L z\right\rangle$ since $L$ maps each $E_{N}$ into itself. Since $w_{N} \rightarrow w^{*}$ weakly, we have

$$
\lim _{N \rightarrow \infty}\left\langle L w_{N}, P_{N} z\right\rangle=\lim _{N \rightarrow \infty}\left\langle w_{N}, L z\right\rangle=\left\langle w^{*}, L z\right\rangle=\left\langle L w^{*}, z\right\rangle .
$$

By (3.3) we have for each $N \in \mathbb{N}$

$$
\left\langle w_{N}, L z\right\rangle=\left\langle L w_{N}, P_{N} z\right\rangle=-\int_{0}^{T}\left[\psi\left(v_{N}\right) q_{N}-g\left(t, u_{N}\right) p_{N}-h(t) p_{N}\right] d t .
$$

Note that $P_{N} z$ is represented by the Fourier series of $z$ truncated to omit the terms associated with the eigenfunctions for the eigenvalues $\left\{\lambda_{n} \mid n \leq-N-1\right\}$. The Fourier series converges to $z$ in $L^{2}\left((0, T), \mathbb{R}^{2}\right)$, so $q_{N} \rightarrow q, p_{N} \rightarrow p$ in $L^{2}((0, T), \mathbb{R})$. Thus

$$
\lim _{N \rightarrow \infty} \int_{0}^{T}\left[\psi\left(v_{N}\right) q_{N}-g\left(t, u_{N}\right) p_{N}-h(t) p_{N}\right] d t=\int_{0}^{T}\left[\psi\left(v^{*}\right) q-g\left(t, u^{*}\right) p-h(t) p\right] d t .
$$

Combining the above, we have

$$
\left\langle L w^{*}, z\right\rangle+\int_{0}^{T}\left[\psi\left(v^{*}\right) q-g\left(t, u^{*}\right) p-h(t) p\right] d t=0
$$

for all $z \in E$, and $w^{*} \in E$ is a weak $T$ periodic solution to (1.3) and, as noted earlier, it is therefore a classical $T$ periodic solution to (1.3) and provides a classical solution to (1.1).

\section{REFERENCES}

[1] H. Brezis and J. Mawhin, Periodic solutions of the forced relativistic pendulum. Differential Integral Equations 23 (2010), no. 9-10, 801-810. MR2675583 (2011h:34084)

[2] J. Mawhin and M. Willem, Critical point theory and Hamiltonian systems. Applied Mathematical Sciences, 74. Springer-Verlag, New York, 1989. MR.982267 (90e:58016) 
[3] P. H. Rabinowitz, Some minimax theorems and applications to nonlinear partial differential equations. Nonlinear analysis, A collection of papers in honor of Erich H. Rothe, ed. by L. Cesari, R. Kannan and H. F. Weinberger, pp. 161-177. Academic Press, New York, 1978. MR $0501092(58: 18545)$

[4] P. H. Rabinowitz, Minimax methods in critical point theory with applications to differential equations. CBMS Regional Conference Series in Mathematics, 65. American Mathematical Society, Providence, RI, 1986. MR845785(87j:58024)

Centro de Modelamiento Matemático and Departamento de Ingeniería Matemática, Universidad de Chile, Casilla 170, Correo 3, Santiago, Chile

E-mail address: manasevi@dim.uchile.cl

Department of Mathematics, The University of Alabama at Birmingham, Birmingham, Alabama 35294

E-mail address: jrward@uab.edu 